\title{
Mitral valve disease, systemic embolism and anticoagulants*
}

\author{
H. A. Fleming \\ M.A., M.D., F.R.C.P. \\ Consultant Cardiologist
}

\author{
Sheila M. BAILEY \\ M.B., B.S. \\ Research Assistant
}

Regional Cardiac Unit, Papworth Hospital, and Addenbrooke's Hospital, Cambridge

\begin{abstract}
Summary
Over a period of $9 \frac{1}{2}$ years patients in East Anglia with mitral valve disease not requiring immediate surgery have been followed.

There has been a high incidence of systemic embolism before the patients are referred.

Long-term treatment with anticoagulants appears to offer a good degree of protection to these patients and, provided the control of the treatment is good, the risks arising from it are small and less than the risks of embolism.
\end{abstract}

SYSTEMIC arterial embolism is a serious complication of mitral disease. The morbidity, the recurrence rate and the mortality are all considerable. Prevention of this disaster is the ideal to be aimed at. Early in the 1960 s, one of the present authors was impressed by the number of patients seen with relatively mild mitral valve disease or a perfectly operable valve, who had yet suffered from disastrous emboli before they were referred. Since then anticoagulants have been used widely in this group of patients in this Unit and since 1962 they have been used in any patient with more than trivial mitral stenosis. A year or two later this usage was extended to mitral incompetence also, provided it was more than trivial. Anticoagulants were used irrespective of the rhythm of the patient. However, new patients with what we believe are preventable emboli are still being seen with some frequency and we feel that this is still a neglected subject.

After 10 years the place of anticoagulants in the prevention of emboli has still not been fully established. In any case the efficiency of anticoagulant control varies greatly from one area to another and it therefore seemed important to determine precisely what the results on this Unit were. In particular answers to the following questions were sought:

(1) How necessary were anticoagulants?

(2) How effective were they?

(3) How safe were they?

(4) Should we be more discriminating in their use?

* Presented to the East Anglian Meeting of the Royal College of Physicians in Cambridge on Saturday, 21 March 1970.

\section{Material}

This is a retrospective study of the cases of mitral valve disease seen through the Regional Cardiac Unit at Papworth in the $9 \frac{1}{2}$ years from the beginning of 1960. The bulk of this report is on 500 such patients who were actually admitted to the unit, and followed for nearly 2000 patient-years to date. Many of these were admitted for the initiation of anticoagulant treatment. In most cases this is now carried out on an out-patient basis. Table 1 shows the composition of the series.

TABLE 1. Mitral valve disease. Content of Papworth series (1970)

\begin{tabular}{ll}
\hline In-patients & 500 \\
Women & $365(73 \%)$ \\
Men & $135(27 \%)$ \\
Age range (years) & $4-79($ average 45$)$ \\
Sinus rhythm & $215(43 \%)$ average \\
& age 39 years \\
Atrial fibrillation & $285(57 \%)$ average \\
& age 50 years \\
Dominant mitral stenosis & $329(66 \%)$ \\
Dominant mitral incompetence & $104(21 \%)$ \\
Mixed lesion, cardiomyopathy & $67(13 \%)$ \\
\hline
\end{tabular}

The usual predominance of women is evident while the age range, the valve pathology and the rhythm would be in keeping with other typical series of mitral valve disease. Mitral myocardiopathy is of the type described by Fleming \& Wood (1959).

\section{Systemic embolization}

Of the 500 cases, $125(25 \%)$ suffered from a systemic embolus. Thirty-seven of these $(30 \%)$ had more than one embolus, the total number of embolic episodes being 173. Twenty-seven per cent of the group were men and $73 \%$ were women. The overall frequency of emboli was $25 \%$ for both men and women. Figure 1 shows the age at the time of the first embolus. It is apparent that while the maximum incidence is between the ages of 40 and 50 years, thirty-one of the patients were under the age of 40 and seven were under the age of 30 years. The increasing embolic incidence with age is associated with a rise in the incidence of atrial fibrillation. The 


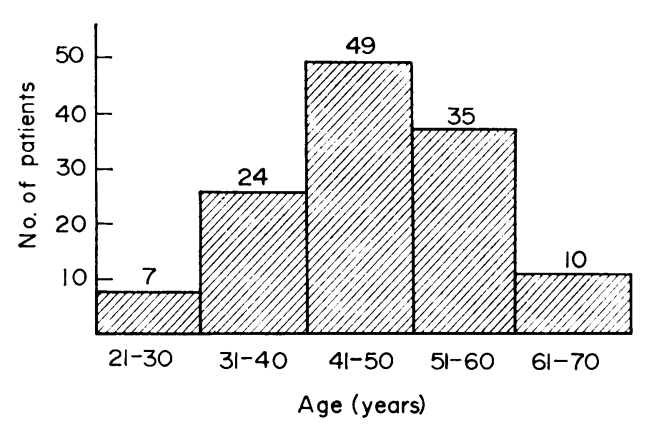

Fig. 1. Mitral valve disease. Distribution of age at time of first embolus.

importance of emboli occurring in the relatively young group is stressed.

\section{Cardiac rhythm}

Twenty-four of the embolic patients were in sinus rhythm. None of these patients gave any history of paroxysmal dysrhythmia. This provides $19 \%$ of the total embolic series and is an incidence of $11 \%$ of the patients in sinus rhythm. In the fibrillating group $35 \%$ suffered emboli. This incidence of patients in sinus rhythm is far from insignificant.

Table 2 shows the age and sex of the embolic patients in sinus rhythm, and indicates that there is a relatively high rate of embolism in young women in sinus rhythm.

TABle 2. Mitral valve disease. Sex and age of twenty-four patients in sinus rhythm

\begin{tabular}{lcccc}
\hline Sex & Number & $\%$ & $\begin{array}{c}\text { Age } \\
\text { range } \\
\text { (years) }\end{array}$ & $\begin{array}{c}\text { Average } \\
\text { age } \\
\text { (years) }\end{array}$ \\
\hline Women & 17 & 71 & $25-60$ & 40 \\
Men & 7 & 29 & $40-61$ & 46 \\
\hline
\end{tabular}

101 Patients with atrial fibrillation similar, but average age 48 years for each sex.

In the out-patient series there are an additional thirty-five embolic patients $20 \%$ of whom were in sinus rhythm. The average age of the fibrillating patients was rather higher at 48 years. In this group the date of the onset of fibrillation in relation to the embolic episode was not always known, but where it was known it appeared that there was a high incidence of emboli within the first year.

\section{Site of the initial embolus}

This is shown in Table 3. These figures are in keeping with other series and again indicate the disturbingly high frequency of cerebral emboli. The incidence of systemic emboli is almost certainly higher than these figures indicate, as small visceral emboli will certainly go unrecognized clinically.
TABLE 3. Site of initial systemic embolus

\begin{tabular}{ll}
\hline Cerebral & $66 \%$ \\
Peripheral & $20 \%$ \\
Visceral & $14 \% *$ \\
\hline
\end{tabular}

* Probably much higher.

\section{Recurrence of emboli}

There were forty-eight embolic recurrences in thirty-seven $(30 \%)$ of the patients. Thirty-five per cent of these occurred within 1 month and $58 \%$ within 12 months of the first episode. The risk of death is much higher with a recurrent rather than an initial embolus.

In this recurrent series only twenty-two of the thirty-seven patients were severe enough to need mitral valvotomy. Three of these patients were in sinus rhythm.

\section{Mitral valve lesion}

Mitral stenosis was the dominant lesion in 116 patients and mitral incompetence in nine. Ten of the patients with recurrent emboli had only trivial or mild valve lesions. Therefore the patient whose lesion is haemodynamically mild is by no means exempt from embolic risk.

\section{Size of the left atrial appendix}

Somerville \& Chambers (1964) suggested that the incidence of embolism was related to the size of the? left atrial appendix. This did not appear to be so in our series. We looked at forty-seven patients in whom a chest X-ray was available within 3 months of embolism and in whom there had been no previous cardiac surgery. The atrial size was graded $0-4$ and the results are shown in Table 4.

TABLE 4. Systemic emboli. Relationship to size of left atrial appendix (LA)

\begin{tabular}{lrrrrr}
\hline & \multicolumn{5}{c}{ Size of LA } \\
\cline { 2 - 6 } & 0 & 1 & 2 & 3 & 4 \\
\hline No. of patients & 5 & 12 & 11 & 17 & 2 \\
Average age (years) & 51 & 48 & 49 & 48 & 45 \\
\hline
\end{tabular}

A considerable number of patients had no marked enlargement of the left atrium or its appendix. This does not appear to be of help in selecting the patients at risk.

\section{The use of anticoagulants}

One hundred and eighteen patients were given anticoagulants to cover surgery and will not be further considered. Two hundred and seventeen patients were treated medically and given anticoagulants on a long-term basis. In this group 
surgery was not considered to be indicated on haemodynamic grounds. We have analysed figures for 649 patient-years of treatment.

Anticoagulants were controlled at widely separated centres in East Anglia. It soon became apparent that the efficiency of control varied considerably from centre to centre and knowledge of the patient's home address was important in deciding whether or not anticoagulants would be indicated.

\section{Complications of anticoagulant treatment}

There was one death in the series. This was from gastro-intestinal haemorrhage and should not have happened. The patient was a woman aged 67 years at the time of death. She had severe pulmonary hypertensive mitral stenosis with three previous major systemic emboli. She declined surgery and cardiac follow-up but she continued to attend the Anticoagulant Clinic for 6 years until her sudden death.

There were eighteen cases of minor bleeding such as vaginal bleeding or epistaxis. It could be considered that these were an aggravation of bleeding rather than actual haemorrhage initiated by anticoagulants.

Details of more significant haemorrhages are given in Table 5.

\begin{tabular}{lr}
$\begin{array}{l}\text { TABLE 5. Haemorrhages on } \\
\text { anticoagulants (649 patient- } \\
\text { years, fourteen cases) }\end{array}$ \\
\hline Renal & 4 \\
Gastro-intestinal & 5 \\
Retinal & 2 \\
Cerebral & 1 \\
Uterine & 1 \\
Pulmonary & 1 \\
Haematoma & 1 \\
Multiple & 1 \\
\hline
\end{tabular}

None of these haemorrhages endangered life. Five of them occurred in cases controlled at one particular centre which had a total of only forty-two treated patients. Thus the complication rate there was rather high. The complication rate for the entire series was $2.3 \%$ per patient-treatment-year. If the small number of patients under the centre of poor control are excluded and the figures analysed since warfarin was used, the complication rate falls to $0.6 \%$ per patient-treatment-year. The death was the only complication occurring in patients with good anticoagulant control.

Hypersensitivity reactions occurred in twelve patients when phenindione was in use. These consisted of skin rashes, diarrhoea, vomiting, and thrombocytopenia needing a change of drug. None of these reactions has occurred since warfarin sodium was used exclusively from 1964 onwards.
In the out-patient series there are an additional seventy patients on treatment with no complications. In most of these anticoagulants were started in 1968 or 1969.

\section{Success of anticoagulant treatment}

There were only five emboli in the 217 patients treated with anticoagulants. This is a rate of $0.8 \%$ per patient-treatment-year. There were no cases of patients on anticoagulants suffering more than one embolus. One case occurred 6 days after the initiation of treatment before full control was achieved, and the other cases occurred from 1 to 6 years after treatment was begun. As far as could be seen the anticoagulant control was adequate in four of these and inadequate in one. This figure for emboli must represent a great reduction on the untreated rate.

In this group, three of the five emboli were cerebral and four of the patients had had emboli before treatment was started. This therefore appears to be a group in which there was a particular embolic risk. There was no other indication of how this group differed from the others.

Six patients were taken off anticoagulants by other doctors and suffered from emboli. A particularly tragic case was that of a girl whom one of us had known from the age of 17 in 1963 when she developed acute rheumatic fever. She quickly developed mitral stenosis and aortic incompetence. She was put on anticoagulants in 1966 but in 1967 she was admitted elsewhere with a miscarriage and anticoagulants were stopped. She was seen again in 1968 , found to be fibrillating and again put on anticoagulants. In 1969 she had haemoptysis and melaena. Her anticoagulants were completely out of control and they were stopped. Five days later she suffered a dense left hemiplegia from which she made no recovery and she died at the age of 22 .

\section{Other illustrative case histories}

\section{Case 1}

Mrs $P$. $P$. aet 46. Symptom-free patient with moderate mixed mitral lesion who was fibrillating but enjoyed all activities, including dancing.

Anticoagulants were advised but this was not carried out and 1 year later, there having been no deterioration in the meantime, she developed a cerebral embolism with dysphasia. There was an incomplete recovery and there has been no recurrence since anticoagulants have been started.

\section{Case 2}

Mrs $H$. B. aet 58. This state-registered nurse had rheumatic fever at the age of 17 . She had been known to have mitral valve disease since 1949 and for many years she had suffered paroxysms of fibrillation. She had only mild mitral valve disease with a late 
opening snap and a short mitral diastolic murmur. She suddenly developed a cerebral embolus which left her very confused, dysphasic and with paresis.

\section{Case 3}

Mrs A. R. aet 31. Mother of three young children. Presented in 1966 with a left retinal artery embolism with poor recovery of vision. Mitral valve disease had been known but she had no disability and on auscultation had only a presystolic murmur, a very late opening snap and a very short mitral diastolic murmur. Chest X-ray showed no more than slight enlargement of the left atrium, and the electrocardiogram a slight $P$ mitrale.

\section{Case 4}

Mrs I. M. aet 44. Had no previous knowledge of rheumatic fever or heart disease until 1962 when she presented with a cerebral embolus. This resulted in a right hemiplegia and aphasia from which there was only a partial recovery. She also had a presystolic murmur, an accentuated first sound and a very short mitral diastolic murmur. Chest X-ray showed no significant enlargement of the left atrium or its appendix, and the electrocardiogram showed only a $P$ mitrale.

\section{Case 5}

Mrs $R$. F. aet 43. Seen in January 1969 with no symptoms. Mild mitral stenosis with atrial fibrillation. Anticoagulants advised but treatment not instituted. March 1969 right femoral embolectomy. Remains well on anticoagulants since.

\section{Discussion}

Systemic embolism is a well recognized complıcation of mitral valve disease. Wood (1954) recorded an incidence of $13 \%$ in his whole series and noted that $75 \%$ of the emboli were cerebral with resultant disastrous effect. In $12.5 \%$ of the embolic cases embolism was the first symptom-often occurring when there was no effort intolerance and the lesion was relatively mild. He also noted that patients with mitral incompetence were not free from emboli and $9.7 \%$ of them suffered emboli. Bannister (1960) followed up 105 of Wood's mitral stenotic patients in whom valvotomy had been deferred because of the mildness of the lesion and he found that twenty-two of these had emboli, fifteen of which were cerebral, five of them causing death. He estimated that for patients under the age of 40 and in sinus rhythm the risk of embolus was $5 \%$.

Fleming \& Wood (1959) separated off a small group of patients with non-critical valve lesions who were fibrillating, with a low cardiac output. This group seemed particularly liable to emboli and longterm anticoagulants were advised.
Marshall (1966) also emphasized hat in mitral stenosis $75 \%$ of the emboli were cerebral and that as many as $12 \%$ may present first because of a cerebral embolus. Further embolization is likely to occur in about $60 \%$ of these patients. The initial mortality is about $7 \%$ and that from further emboli is about $27 \%$. He says, 'although the exact place of anticoagulant therapy in the management of cerebral vascular disease is still the subject of some disagreement, there is practically universal acquiescence in its value in cases of cerebral embolization'. He suggests that ideally treatment should be begun before the first embolus occurs. This is the ideal that has been aimed at in the present series.

The high incidence of epilepsy and hypertension in patients with mitral valve disease has suggested that small emboli with infarction are even more frequent than major clinical episodes would indicate. Obeyesekere et al. (1965) found a high incidence of renal infarction in a necropsy series. Other studies also suggest that the obvious embolic episodes are just the visible tip of an iceberg. Hutchinson \& Stock (1963) studied paroxysmal cerebral ischaemia in patients with rheumatic heart disease. Three hundred and twenty-three such patients were reviewed for neurological symptoms and it was found that there had been sixty classical cerebrak embolic episodes in forty-four patients $(14 \%$ of the series). Thirty-eight patients $(12 \%)$ gave a history of disabling recurrent neurological disturbances. These attacks were not uncommon in patients with sinus? rhythm.

Swash \& Earl (1970) particularly sought a history of transient visual obscuration and found that $33 \%$ were patients with rheumatic heart disease. None of these spontaneously complained of symptoms. They seemed to be embolic in nature, possibly from platelet aggregation. Thirty-five percent of the patients in atrial fibrillation and $30 \%$ in sinus rhythm had the symptoms. Anticoagulant treatment appeared to diminish the incidence. Cleland et al. (1969) noted that embolism is common in the absence of prophylactic treatment and they found an incidence of $42 \%$ in 155 patients followed for 10 years. They mention that it usually occurs in the presence of atrial fibrillation but may be found in sinus rhythm.

Coulshed et al. (1970) reported a very detailed study of systemic embolism in mitral valve disease dealing with 737 cases of mitral stenosis and 102 cases of mitral incompetence. The embolic incidence increased with age and with atrial fibrillation but was not related to disability. In mitral stenosis with sinus rhythm there was an $8 \%$ incidence and in atrial fibrillation it was $31.5 \%$. In mitral incompetence in sinus rhythm the incidence was $0.7 \%$ and in atrial fibrillation $22 \%$. Of the 157 with emboli $20 \%$ were in 
sinus rhythm. In 166 non-surgical patients with mitral stenosis the embolic incidence was $3.7 \%$ per patient-year with seven embolic deaths. Multiple emboli occurred in seventy-nine patients, twenty-four having more than one recurrence. Seven of these were in sinus rhythm. They note particularly that embolism is as common in mitral incompetence as in mitral stenosis, and where sinus rhythm is present valvotomy has no effect on the embolic incidence. Also, unless valvotomy was indicated on haemodynamic grounds, it had no beneficial effect on the incidence of emboli.

Is there any way of selecting patients who are particularly at risk? Somerville \& Chambers (1964) suggested that the incidence of embolism is directly related to the size of the left atrium and particularly its appendix. However, a number of other studies such as the present one and that of Coulshed et al. (1970) have failed to verify this. In any case, $14 \%$ of their embolic series had a small left atrial appendix.

While statistically age, atrial fibrillation, size of the left atrium and appendix, low cardiac output and cardiomegaly increase the risk of embolism many of the patients will fall outside these parameters. It therefore seemed that all patients with more than trivial mitral valve disease should be considered for protection without waiting for emboli to occur.

The administration of anticoagulants on a longterm basis seemed the only possibility. Wood \& Conn (1954) describe seven embolic cases of mitral stenosis they treated. They found that emboli recurred when treatment was withdrawn and suggested that it should be continued indefinitely. Askey \& Bernstein (1960) emphasize that while $25-30 \%$ of adults with rheumatic heart disease die of embolism there is an even greater incidence of disability. Again they report that embolism is frequently the first sign in an otherwise well patient. They suggest that moderate treatment with anticoagulants decreases this risk. Lillicrap \& Piesowicz (1964) made a similar suggestion, also noting that the occurrence of embolism is not an additional reason for mitral valvotomy. Szekely (1964) reported a very thorough study of 754 patients with chronic rheumatic heart disease followed over a period of 5833 patient-years. He noted that emboli were seven times more common in atrial fibrillation than in sinus rhythm and that where the duration of the fibrillation was known one-third of the emboli occurred within 1 month and two-thirds within 12 months of the onset of fibrillation. The incidence of embolic recurrences was $8 \%$ per patient-year irrespective of the cardiac rhythm. Mitral valvotomy did not eliminate the danger of embolism. It was questionable whether it reduced its incidence. He found that anticoagulants reduced the incidence of embolic recurrences and suggested that it should be continued for 1 year after the onset of atrial fibrillation or after an embolic episode and probably for much longer periods in selected cases. Long-term treatment should also be considered in patients after the first embolic episode, both in sinus rhythm and in atrial fibrillation.

Casella, Abelmann \& Ellis (1964) also report emboli in asymptomatic patients with mild valve disease and tentatively suggest that long term anticoagulants may be a method of controlling this. They suggested that control studies are badly needed. While agreeing with this it seems unlikely that these studies will now be carried out. The reduced incidence of this type of patient in the Western world makes this even less likely. The authors feel that if tragic cases are to be avoided all patients at any risk should be considered for treatment. The type of left atrium with multiple diverticula shown in Fig. 2 makes it not surprising that thrombus and embolism should be so liable to occur. This was a 45-year-old woman in sinus rhythm with a mild mixed mitral lesion with dominant stenosis. In the PA chest radiograph there was insignificant enlargement of the left atrium. In the lateral angiocardiogram the diverticula

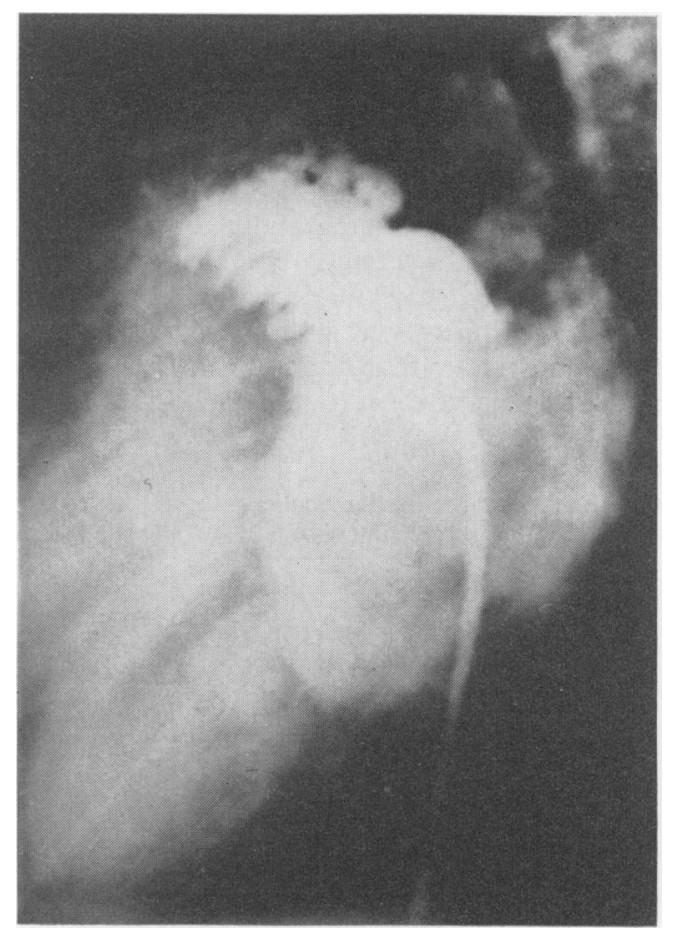

FIG. 2. Left lateral angiocardiogram. Brockenbrough technique. Injection into the left atrium showing numerous diverticula arising from the atrial appendix. Woman aged 45 years in sinus rhythm with mild mixed mitral valve lesion. 
are well seen. It is easy to imagine how imperfectly these empty where there is any degree of stagnation in the left atrium and it is also obvious that with the onset of atrial fibrillation the loss of atrial systole will greatly aggravate this situation and the liability to clot formation.

This study emphasizes that emboli can occur in young symptom-free patients in sinus rhythm with mild mitral valve disease, and that long term anticoagulant treatment can be used to reduce the incidence of emboli with little resultant risk to the patient provided the control of the treatment is carried out with due care.

It is disturbing to find that patients are still having emboli before they are referred and that advice about anticoagulants (as in Case 5) is not always accepted by the referring physician. We therefore consider that anticoagulants are very necessary. That they are effective is indicated by the fact that in the $9 \frac{1}{2}$-year period of the study only five emboli occurred in the medical cases of mitral valve disease who were on anticoagulants. The relative safety is indicated by the lack of serious complications, and a minor complication rate of $2.3 \%$ per patient-treatment-year over the whole series and $0.2 \%$ with good control on warfarin.

Finally we consider that we should not be more restricted in our use of anticoagulants to patients with more than trivial mitral valve disease. Youth, sinus rhythm and a small left atrium offer no absolute protection against embolus. The greatest difficulty arises in deciding about the milder cases. and in these other aspects of the patient's life or personality may sway the decision. Patients with a simple mitral presystolic murmur or with a mitral late or pansystolic murmur have not been treated, but most others have.

\section{Acknowledgment}

Our thanks are due to many colleagues who have referred patients and answered our queries, to the pathological laboratories who have carried out the blood tests, and to the East Anglian Regional Hospital Board who provided a research grant for S.M.B.

\section{References}

Askey, J.M. \& Bernstein, S. (1960) The management of rheumatic heart disease in relation to systemic arterial embolus. Progress in Cardiovascular Diseases, 3, 220.

BANNISTER, R.G. (1960) The risk of deferring valvotomy in patients with moderate mitral stenosis. Lancet, ii, 325.

Casella, L., Abelmann, W.H. \& Ellis, L.B. (1964) Patients with mitral stenosis and systemic emboli. Haemodynamic and clinical observation. Archives of Internal Medicine, 114, 773.

Cleland, W., Goodwin, J., McDonald, L. \& Ross, D. (1969) Medical and Surgical Cardiology, p. 847. Blackwell Scientific Publications, Oxford.

Coulshed, N., Epstein, E.J., MacKendrick, C.S., GalloWAY, R.W. \& WALKER, E. (1970) Systemic embolism in mitral valve disease. British Heart Journal, 32, 26.

Fleming, H.A. \& Wood, P.H. (1959) The myocardial factor in mitral valve disease. British Heart Journal, 21, 117.

Hutchinson, E.C. \& Stock, J.P.P. (1963) Paroxysmal cerebral ischaemia in rheumatic heart disease. Lancet, ii, 653.

Lillicrap, David \& Peisowicz, Alina (1964) Mitral stenosis and systemic emboli. British Medical Journal, 2, 1169.

Marshall, John (1966) The prevention of strokes. American Heart Journal, 71, 1.

Obeyesekere, H.I., Dulake, M., Dermerdash, H. \& Hollister, R. (1965) Systemic hypertension and mitra valve disease. British Medical Journal, 2, 441.

Somerville, W. \& Chambers, R.J. (1964) Systemic embolism in mitral stenosis: relation to the size of the left atrial appendix. British Medical Journal, 2, 1167.

Swash, Michael \& EARL, C.J. (1970) Transient visual obscurations in chronic rheumatic heart disease. Lancet, ii, 323.

Szekely, Paul (1964) Systemic embolism and anticoagulant prophylaxis in rheumatic heart disease. British Medical Journal, 1, 1209.

Wood, J.C. \& ConN, H.L. (1954) Prevention of systemic arterial embolism in chronic rheumatic heart disease by means of protracted anticoagulant therapy. Circulation, $10,517$.

WooD, P.H. (1954) An appreciation of mitral stenosis. Part I. Clinical features. British Medical Journal, 1, 1051. 